\title{
Stopping While Going! Response Inhibition Does Not Suffer Dual-Task Interference
}

\author{
Motonori Yamaguchi, Gordon D. Logan, and Patrick G. Bissett \\ Vanderbilt University
}

\begin{abstract}
Although dual-task interference is ubiquitous in a variety of task domains, stop-signal studies suggest that response inhibition is not subject to such interference. Nevertheless, no study has directly examined stopsignal performance in a dual-task setting. In two experiments, stop-signal performance was examined in a psychological refractory period task, in which subjects inhibited one response while still executing the other. The results showed little evidence for the refractory effect in stop-signal reaction time, and stop-signal reaction time was similar in dual-task and single-task conditions, despite the fact that overt reaction times were significantly affected by dual-task interference. Therefore, the present study supports the claim that response inhibition does not suffer dual-task interference.
\end{abstract}

Keywords: response inhibition, executive control, psychological refractory period, horse-race model, stop-signal paradigm

The ability to inhibit inappropriate thoughts and actions constitutes a major class of executive control capabilities. Inhibitory processing is believed to play a central role in exercising cognitive control in everyday activities (e.g., Hasher \& Zacks, 1988). Impairment of inhibitory control is related to a variety of psychological and neurological disorders (e.g., Aron \& Poldrack, 2005; Chamberlain et al., 2006; Enticott, Ogloff, \& Bradshaw, 2008; Logan, Schachar, \& Tannock, 2000). Therefore, understanding of the nature of inhibitory processes has been of major interest to psychologists and neuroscientists. One of the experimental paradigms used to study executive control and inhibitory processing is the stop-signal paradigm (Logan, 1994; Logan \& Cowan, 1984; Verbruggen \& Logan, 2008), which offers a means to explicitly test the ability to inhibit responses when the responses are being prepared or have already been prepared (De Jong, Coles, Logan, \& Gratton, 1990).

In a stop-signal task, subjects are instructed to perform a go task, such as simple- or choice-reaction tasks (Lappin \& Eriksen, 1966; Logan, Cowan, \& Davis, 1984; Ollman, 1973), or more complex tasks such as type writing (Logan, 1982) and baseball batting (Gray, 2009). In a proportion of trials (stop-signal trials), the go-task stimulus is followed by a stop signal, and subjects are required to withhold, or inhibit, responses on these trials. A stop-signal trial is called a signalinhibit trial if subjects successfully inhibit their response, whereas it is called a signal-respond trial if subjects fail to do so. A robust finding is that subjects have more difficulty inhibiting responses when the

This article was published Online First May 16, 2011.

Motonori Yamaguchi, Gordon D. Logan, and Patrick G. Bissett, Department of Psychology, Vanderbilt University.

The research was supported by Grant BCS 0957074 from the National Science Foundation and Grant number 2R01 EY008890-20 from the National Eye Institute.

Correspondence concerning this article should be addressed to Motonori Yamaguchi, Department of Psychology, Vanderbilt University, Nashville, TN 37203. E-mail: motonori.yamaguchi@ vanderbilt.edu interval between the go-task stimulus and the stop signal (stop-signal delay or SSD) increases.

Much like other cognitive functioning, which is studied primarily by measuring how long it takes to produce a response when a stimulus is presented (i.e., reaction time or RT), stop-signal performance is also examined by measuring how long it takes to inhibit a response when a stop signal is presented, which is called stop-signal reaction time (SSRT). Because response inhibition is implicit, SSRT is not directly measurable. However, by applying the horse-race model (Logan \& Cowan, 1984), SSRT can be computed based on other observable performance measures.

According to the horse-race model, the go-task (GO) process races against an inhibitory (STOP) process. The response is inhibited if the STOP process completes before the GO process (see Figure 1a). This occurs when RT is longer than the sum of SSD and SSRT. That is, a signal-inhibit trial occurs if RT $>$ SSD + SSRT. On the other hand, response is executed (stopping fails) if the GO process completes before the STOP process. Hence, a signal-respond trial occurs if RT $\leq$ SSD + SSRT. Therefore, the proportion of signal-respond trials is an estimate of the probability that RT is shorter than the sum of SSD and SSRT; that is, $p($ RespondlSignal $)=\operatorname{Pr}[\mathrm{RT} \leq \mathrm{SSD}+\mathrm{SSRT}]$. This probability corresponds to the area under the RT density function to the left of the value that equals SSD plus SSRT (see the shaded area in Figure 1b). Thus, SSRT can be computed if the RT distribution and SSD are known. In practice, this integration method amounts to subtracting SSD from go-trial RT at the percentile corresponding to $p$ (RespondlSignal) (see, Logan, 1994, pp. 215-216). ${ }^{1}$

\footnotetext{
${ }^{1}$ To provide a more concrete example of how SSRT is computed based on the integration method, let us assume $p($ Respond $\mid$ Signal $)=0.4$ at $\mathrm{SSD}=300 \mathrm{~ms}$. RT for go trials at $p$ (Respond $\mid$ Signal $)=0.4$ is the 40-percentile RT (the value below which $40 \%$ of all RTs fall), which may be $500 \mathrm{~ms}$. Then, SSRT is equal to $500-300=200 \mathrm{~ms}$.
} 

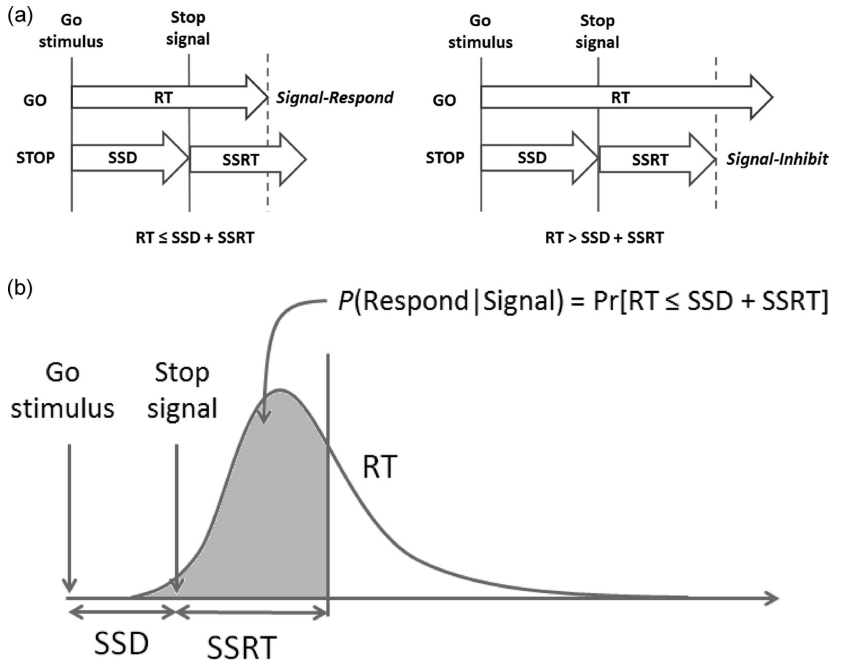

Figure 1. Schematic illustrations of the horse-race model.

\section{Dual-Task Interference in Stop-Signal Performance?}

The stop-signal task is structurally similar to the psychological refractory period (PRP) task (Telford, 1931; Welford, 1952): Both tasks involve two successive stimuli and distinct responses to each stimulus. Hence, two different processes presumably operate concurrently in both task conditions. However, outcomes of the two procedures are in sharp contrast.

A robust finding in the PRP paradigm is that the response to the second of two successive stimuli is slower when the interval between these stimuli (stimulus onset asynchrony, or SOA) is short than when it is long. This dual-task interference, or PRP effect, is found in a wide range of tasks (Pashler, 1994). Although there have been reports suggesting that perfect or nearly perfect timesharing is possible between two concurrent tasks under certain conditions (Greenwald \& Shulman, 1973; Hazeltine, Teague, \& Ivry, 2002; Schumacher et al., 2001), these results are not always replicated (Levy \& Pashler, 2001; Lien \& Proctor, 2002; Shin, Cho, Lien, \& Proctor, 2007; Tombu \& Jolicœur, 2004) and the PRP effect typically persists even after extensive training (Gottsdanker \& Stelmach, 1971; Ruthruff, Johnston, \& Van Selst, 2001; Van Selst, Ruthruff, \& Johnston, 1999).

Unlike PRP studies, stop-signal studies suggest that two concurrent processes can proceed without much interference (e.g., Logan, 1981; Logan \& Burkell, 1986; Logan et al., 1984). For instance, Logan (1981) manipulated stimulus eccentricity and stimulus-response compatibility in the go task. Whereas RT varied according to these manipulations, SSRT was virtually unaffected, suggesting that response inhibition is free from influence of the go-task demands. Likewise, while RT is dynamically adjusted according to the probability of stop-signal occurrences, SSRT is largely unaffected by such manipulations (Bissett \& Logan, 2011; Logan, 1981; Verbruggen \& Logan, 2009b). These findings suggest that STOP and GO processes depend on different factors.

Logan and Burkell (1986) provide a more direct comparison of dual-task and stop-signal performances. In their study, subjects performed letter discrimination. In a portion of trials, a letter was followed by a tone with various delays. The stop-signal group responded to the tone by withholding the response to the letter; the dual-task group responded to the tone by pressing another key without inhibiting the response to the letter. The dual-task group showed a typical PRP effect in the second response; RT increased as the delay of tone decreased. In contrast, the stop-signal group showed little evidence for the PRP effect; SSRT was not affected much by the delay. Logan and Burkell showed that the pattern of SSRT obtained in the stop-signal group was similar to that predicted by the horse-race model that assumed independence of GO and STOP processes. Therefore, the STOP process was not subject to the same refractory effect as the overt responses in the dual-task condition.

\section{The Present Study}

Given that the PRP effect is ubiquitous in a variety of cognitive tasks, the lack of dual-task interference in the stop-signal procedure is intriguing. However, no study has actually examined stopsignal performance in a dual-task setting. Therefore, the present study carried out a stringent test of stop-signal performance in dual-task contexts. In the present study, the go task was a PRP task in which subjects performed two choice-reaction tasks to two successive visual stimuli. In a portion of trials, subjects were also presented with a tone that served as a stop signal and were asked to inhibit one response without inhibiting the other response. Thus, subjects had to perform response execution and response inhibition in stop-signal trials.

In Experiment 1, the main focus was on whether SSRT is subject to the PRP effect. Each subject was tested in three conditions. In the first condition (Stop-R1), subjects were asked to inhibit the first response (R1) and execute the second (R2) when a stop signal occurred. In the second condition (Stop-R2), they were asked to inhibit R2 and execute R1. In the third condition (NoStop), no stop signals were presented, and subjects were asked to execute both R1 and R2. As with go RTs, the possibility of a PRP effect on SSRT can be assessed by comparing SSRT at short and long SOAs. If the STOP process shares the same bottleneck with the GO1 and GO2 processes, then SSRT should be longer at short SOAs when the STOP process overlaps with the bottleneck process in GO1 or GO2 than at long SOAs when the STOP process does not overlap with the bottleneck processes in GO1 and GO2. This should occur in both the Stop-R1 and Stop-R2 condition. However, if the STOP process does not share the same bottleneck with the GO1 and GO2 processes, then SSRT should be unaffected by SOA.

Experiment 2 was conducted to obtain converging evidence by comparing SSRTs in single-task and dual-task conditions. Subjects performed a single-task condition for which only S1 or $\mathrm{S} 2$ occurred, as well as a dual-task condition (either the Stop-R1 or Stop-R2 condition identical with those in Experiment 1). As typically observed, the go-task RT should be longer in the dual-task condition than in the single-task condition, because of increased task load in the dual-task condition (e.g., Logan \& Gordon, 2001). If the STOP process is subject to the same dual-task interference as the GO process, then SSRT should also be longer in the dual-task condition than in the single-task condition. 


\section{Experiment 1}

\section{Method}

Participants. Twenty subjects were recruited from the Vanderbilt University community. Six subjects were paid $\$ 12$ for their participation, and the remaining subjects participated for partial fulfillment of their psychology courses. All reported having normal or corrected-to-normal visual acuity, normal color vision, and normal hearing.

Apparatus and stimuli. The apparatus consisted of a 19-in. VGA monitor and a personal computer. The experiment was controlled by an E-Prime program (Psychology Software Tools, Sharpsburg, PA). Responses were registered by pressing keys ( $Z$, $X, N$, and $M$ ) on a QWERTY keyboard. Stimuli were red and green circles $(2.0 \mathrm{~cm}$ in diameter), black squares $(1.5 \mathrm{~cm}$ in sides $)$, and black diamonds ( $1.5 \mathrm{~cm}$ in sides), presented against a white background. The stop signal was a sinusoidal tone $(500 \mathrm{~Hz}, 50 \mathrm{~dB})$ binaurally presented through headphones, which lasted for $100 \mathrm{~ms}$.

Procedure. The experiment was conducted individually in a room under normal fluorescent lighting. Subjects sat in front of the computer monitor and read on-screen instructions. An experimental session consisted of a practice phase, in which subjects performed one 6-trial block of Task1, one 6-trial block of Task2, and one 24-trial block of the dual-task condition. Responses for Task1 (R1) were made by pressing the $Z$ and $X$ keys with the middle and index fingers of the left hand, respectively, and responses for Task2 (R2) were made by pressing the $N$ and $M$ keys with the index and middle fingers of the right hand, respectively. For half the subjects, Task1 was shape discrimination, and Task 2 was color discrimination; Task1 and Task2 were switched for the other half. For the color task, subjects pressed one key to red circles and the other key to green circles. For the shape task, subjects pressed one key to squares and the other key to diamonds. The stimulusresponse mappings were counterbalanced across subjects.

After the initial three practice blocks, subjects went on to two additional practice blocks for the two stop-signal conditions in which go and stop-signal trials appeared in random order. They were given one block of practice trials in the Stop-R1 condition, in which subjects inhibited R1, and one block in the Stop-R2 condition, in which they inhibited R2. The order of these practice blocks was counterbalanced across subjects. Each practice block consisted of 24 trials, one third of which were randomly chosen to be stop trials and SSD was held at $250 \mathrm{~ms}$ throughout the practice blocks.

Following the practice phase was a test phase, which consisted of two cycles of three blocks. Each cycle consisted of one Stop-R1 block, one Stop-R2 block, and one No-Stop block. The former two conditions were composed of 72 go trials and 36 stop-signal trials (i.e., one third of trials were stop-signal trials), and the No-Stop condition consisted of 72 go trials. The order of the three conditions was counterbalanced across participants using a Latin square, and the order was retained for the second cycle. Subjects were informed at the beginning of each block as to which condition they were about to perform. Subjects initiated each block by pressing the space bar.

The sequences of stop-signal and go trials are illustrated in Figure $2 \mathrm{a}$ and $2 \mathrm{~b}$, respectively. A go trial started with a fixation cross $(0.8 \mathrm{~cm}$ in width and height) at the center of screen, which lasted for $500 \mathrm{~ms}$. The imperative stimulus for Task1 (S1) occurred above the cross at a center-to-center distance of $2.6 \mathrm{~cm}$. With a variable SOA $(100,300$, or $900 \mathrm{~ms})$, the imperative stimulus for Task2 (S2) occurred below the cross. The stimuli remained on the screen until a response to S2 was made or for 3,000 ms after S2 onset. Feedback for Task1 and Task 2 appeared at the respective stimulus locations. The Feedback was the message "CORRECT!" for a correct response, "WRONG!" for an error response, and "TOO SLOW!" for no response. The feedback display lasted for 1,000 ms if both responses were correct and 5,000 ms otherwise.

The sequence for a stop-signal trial was essentially the same as that of a go trial, except that a stop signal occurred sometime during the trial. When the stop signal occurred, subjects withheld $\mathrm{R} 1$ or R2, according to the condition, until the trial ended. SSD was the interval between the imperative stimulus (S1 or S2 depending on the to-be-inhibited response) and a stop signal. SSD was initially set at $250 \mathrm{~ms}$ for both conditions and adjusted according to subjects' performance using a tracking procedure:

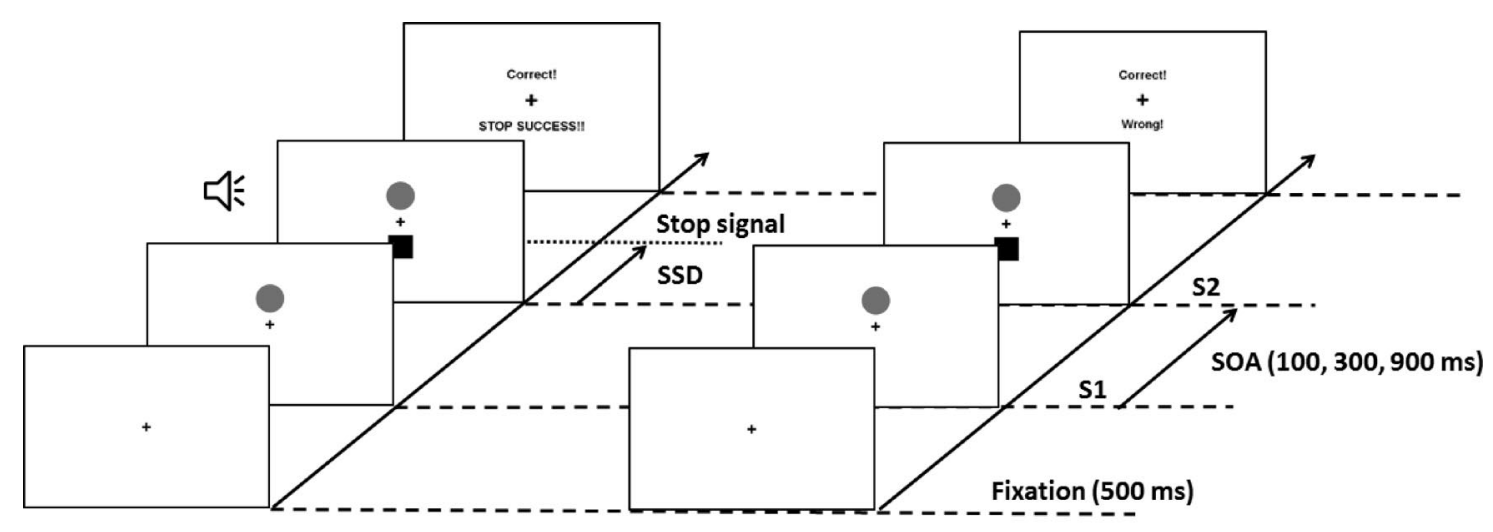

(a) Stop-signal trial

(b) Go trial

Figure 2. Illustrations of the sequences of (a) stop-signal and (b) go trials (an example shows the Stop-R2 condition) of Experiment 1. 
SSD was incremented by $50 \mathrm{~ms}$ when subjects successfully stopped responding and decremented by $50 \mathrm{~ms}$ when subjects failed to stop responding. The lower and upper limits of SSD were $-500 \mathrm{~ms}$ and 2,900 ms, respectively. SSD was tracked separately for the three SOAs and for the Stop-R1 and Stop-R2 conditions. Feedback was "STOP FAILED!" when subjects failed to stop a response and "STOP SUCCESS!" when they successfully stopped a response. Subjects were told that, on some trials, a stop-signal would occur so late that it would be impossible to stop responding, so they should not slow responses to wait for a signal. Instead, the experimenter emphasized that subjects should try to respond to the go stimuli as quickly and as accurately as they could and to try to stop responding when they heard a tone.

\section{Results}

Overt RT. Trials for which RT1 and/or RT2 were $<200$ or $>2000$ were discarded (2.47\% of all trials). Percentages of errors were computed for Task1 and Task2 (see Table 1), but no significant effect was revealed for these measures. Thus, our discussions focus on the RT data. Mean RT1 and RT2 for trials for which R1 and R2 were both correct were computed for each subject and were submitted to separate analysis of variance (ANOVA; see Table 2). The results are summarized in Figure 3.

The No-Stop condition yielded typical outcomes of the PRP paradigm: R2 was faster for long SOAs than for short SOAs, yielding the PRP effect, but SOA had little influence on RT1. The outcomes were similar in the Stop-R1 and Stop-R2 conditions.

In the Stop-R1 condition, RT1 tended to be shorter for signalrespond trials $(M=626 \mathrm{~ms})$ than for go trials $(M=801 \mathrm{~ms})$, which is in accord with the horse-race model; signal-respond trials are those for which the GO1 process completed quickly and escaped inhibition (i.e., RT1 $\leq$ SSD + SSRT; Logan \& Cowan, 1984). On the other hand, RT2 tended to be longer for the two types of stop-signal trials (911 ms for signal-inhibit trials, and 866 $\mathrm{ms}$ for signal-respond trials) than for go trials $(793 \mathrm{~ms})$. This slowing of R2 in stop-signal trials likely reflects post-stop-signal slowing (i.e., the prolongation of go RT on trials that immediately follow stop-signal trials; Bissett \& Logan, 2011; Rieger \& Gauggel, 1999; Verbruggen, Logan, Liefooghe, \& Vandierendonck, 2008). The greater slowing after signal-inhibit trials may have occurred because signal-inhibit trials tend to occur when the GO1 process is slow (i.e., RT1 $>$ SSD + SSRT), so the GO2 process may have been queued longer. Consistent with this interpretation, the slowing of R2 dissipated at long SOAs, for which Task1 processing had little influence on RT2.

In the Stop-R2 condition, RT2 was generally shorter for signalrespond trials $(M=644 \mathrm{~ms})$ than for go trials $(M=779 \mathrm{~ms})$, and the PRP effect was statistically similar for the two trial types. In addition, RT1 was shortest for signal-respond trials $(M=589 \mathrm{~ms})$, intermediate for go trials $(M=663 \mathrm{~ms})$, and longest for signalinhibit trials $(M=752 \mathrm{~ms})$. These results reflect a tendency for RT1 and RT2 to covary in a PRP task (e.g., Way \& Gottsdanker, 1968): signal-respond trials tend to occur when RT2 (and, because of the correlation, RT1) is shorter, whereas signal-inhibit trials tend to occur when RT2 (and RT1) is longer. The RT1 differences were particularly large at short SOAs and disappeared at long SOAs, reflecting the fact that the correlation between RT1 and
RT2 tends to be strong at short SOAs but disappears at long SOAs (e.g., Way \& Gottsdanker, 1968).

As in typical PRP studies, the PRP effects were obtained in all conditions. The size of the PRP effect (difference between the 100and 900-ms SOAs) was on the order of 300-450 $\mathrm{ms}$ in the Stop-R1 condition and 250-300 ms in the Stop-R2 condition. In all three conditions, the RT1 and RT2 data conform to the predictions of the horse-race model; responses were faster for signalrespond trials and slower for signal-inhibit trials than go trials.

Stop-signal RT. Mean SSD and the probability of responding in stop-signal trials, $p$ (RespondlSignal), were computed for each SOA in the Stop-R1 and Stop-R2 conditions (see Table 3). Although a tracking algorithm was used to adjust SSDs, the values of $p$ (RespondlSignal) were different from .5 in all conditions, perhaps because the initial SSD values were too small and allowed subjects to stop responding more often than they should have in the early trials. Consequently, SSRT was computed by using the integration method (Logan, 1994), which can be used for any value of $p$ (RespondlSignal). That is, SSRT was obtained by subtracting mean SSD from RT in go trials (without stop signals) at the percentile of the go RT distribution that corresponds to $p$ (RespondlSignal) (see Figure 1b).

The mean SSRTs across subjects are plotted as a function of SOA for Stop-R1 and Stop-R2 conditions in Figure 4. SSRT was short $(M=255 \mathrm{~ms})$ and unaffected by SOA, showing no PRP effect. An ANOVA as a function of Condition and SOA confirmed this impression; neither the main effects nor their interaction approached significance (see Table 4). SSRT was similar to the value obtained in prior studies for which the go task was a single task (e.g., Logan \& Cowan, 1984). Collectively, there is no evidence for dual-task interference in stop-signal performance.

\section{Discussion}

The results of the present experiment are clear-cut. The PRP effect was obtained in the RT2 data of all conditions, suggesting $\mathrm{GO} 1$ and GO2 processes are functionally dependent. The RT data also indicated that responses were generally faster for signalrespond trials and slower for signal-inhibit trials than go trials. These outcomes reflect the variability inherent in task performance (see Nelson et al., 2010), and the results are consistent with the horse-race model, according to which signal-respond (-inhibit) trials occur when RT is shorter (longer) than the sum of SSD and SSRT.

More importantly, the present experiment showed that SSRT was not affected by SOA in the Stop-R1 or Stop-R2 conditions. The outcomes are consistent with the assumption of functional independence between GO and STOP processes. SSD increased as SOA decreased in the Stop-R2 condition. Provided that the STOP process is not subject to the PRP effect, however, this is a necessary consequence of the SSD tracking algorithm: RT2 changes with SOA, but SSRT is relatively constant. Thus, SSD must change with RT to obtain a specific value of $p$ (RespondlSignal). Therefore, the present results are consistent with prior observations that the STOP process is independent of the go-task demands (e.g., Logan, 1981; Logan \& Cowan, 1984).

It should also be noted that the SSRTs in Experiment 1 were similar to those that are typically observed in single-task experiments (e.g., Logan \& Cowan, 1984), but the present experiment 
Table 1

Percentages of Errors for Task1 (PE1) and Task2 (PE2) for Go Trials of the No-Stop, Stop-R1, and Stop-R2 Conditions in Experiment 1 as a Function of SOA

\begin{tabular}{cccccccc}
\hline & \multicolumn{3}{c}{ PE1 } & & \multicolumn{3}{c}{ PE2 } \\
\cline { 2 - 3 } \cline { 6 - 7 } SOA & No-Stop & Stop-R1 & Stop-R2 & & No-Stop & Stop-R1 & Stop-R2 \\
\hline 100 & 2.33 & 2.27 & 2.69 & & 5.79 & 6.71 & 3.88 \\
300 & 2.32 & 2.93 & 1.58 & & 6.56 & 6.47 & 4.28 \\
900 & 2.21 & 3.04 & 2.45 & & 4.94 & 6.68 & 3.53 \\
\hline
\end{tabular}

lacks an appropriate control condition to evaluate this comparison. To obtain further evidence for the lack of dual-task interference in SSRT, Experiment 2 directly compared stop-signal performance in dual- and single-task conditions.

\section{Experiment 2}

\section{Method}

Participants. Forty subjects were newly recruited from the same population as in Experiment 1, with the same criteria applied for subject selection. Eighteen subjects were paid $\$ 12$, and the remaining subjects received course credits for their psychology courses for participation. Half the subjects were randomly assigned to the Stop-R1 group, and the remaining subjects were assigned to the Stop-R2 group.

Apparatus, stimuli, and procedure. The apparatus and stimuli were identical with those used in Experiment 1. The procedure closely followed Experiment 1, except for the following differences: All subjects performed eight trial blocks: two cycles each consisting of three dual-task blocks and one single-task block. The dual-task blocks were identical with the Stop-R1 or Stop-R2 conditions of Experiment 1, depending on the subject group. The single-task block differed from the dual-task block in the respect that a single target stimulus appeared on each trial; for the Stop-R1 group, the target stimulus was always S1 and S2 never appeared; and for the Stop-R2 group, the target stimulus was always S2 and S1 never appeared (i.e., S2 immediately followed the fixation screen). Subjects were informed at the beginning of each block as to whether the current block was a single- or dual-task condition. Both the single- and dual-task blocks consisted of 72 trials, of which one third were stop-signal trials. Thus, there was a total of 144 trials (96 go trials and 48 stop-signal trials) for each SOA (100, 300, $900 \mathrm{~ms}$, and single-task).

SSRT is estimated most reliably when $p$ (RespondlSignal) is close to .5 (Band, van der Molen, \& Logan, 2003). However, in Experiment 1, $p$ (RespondlSignal) was smaller than .5 for all conditions, possibly because the initial SSD $(250 \mathrm{~ms})$ was too small as compared to mean SSD in the respective conditions. Thus, to start the present experiment with SSDs that would produce $p$ (RespondlSignal) close to .5 , initial SSDs were determined as follows: First, median RTs for the go trials were computed for the three SOAs of the Stop-R1 and Stop-R2 conditions of Experiment 1 , and mean SSRTs of the respective conditions were subtracted from the medians. The resulting values correspond to mean SSDs that would have produced $p$ (RespondlSignal $)=.5$ in Experiment 1. This procedure yielded mean SSDs of 436, 487, and $479 \mathrm{~ms}$ for the 100-, 300-, and 900-ms SOAs of the Stop-R1 trials, and 600, 433, and $338 \mathrm{~ms}$ for the 100-, 300-, and 900-ms SOAs of the Stop-R2 trials. Thus, the initial SSDs were set at $450 \mathrm{~ms}$ for the three SOAs for the Stop-R1 group and at 600,450 , and $350 \mathrm{~ms}$ for

Table 2

ANOVA for Overt Reaction Times for Task1 (RT1) and Task2 (RT2) for the No-Stop, Stop-R1, and Stop-R2 Conditions of Experiment 1

\begin{tabular}{|c|c|c|c|c|c|c|c|c|}
\hline \multirow[b]{2}{*}{ Factor } & \multicolumn{4}{|c|}{ RT1 } & \multicolumn{4}{|c|}{ RT2 } \\
\hline & $F$ & $d f$ & $M S E$ & $\eta_{p}^{2}$ & $F$ & $d f$ & $M S E$ & $\eta_{p}^{2}$ \\
\hline \multicolumn{9}{|l|}{ No-Stop Condition } \\
\hline $\mathrm{SOA}^{\mathrm{a}}$ & $<1$ & 2,38 & 1,673 & .030 & $155.38^{* *}$ & 2,38 & 2,547 & .891 \\
\hline \multicolumn{9}{|l|}{ Stop-R1 Condition } \\
\hline Trial Type ${ }^{\mathrm{b}}$ & $76.83^{* *}$ & 1,19 & 11,904 & .802 & $11.36^{* *}$ & 2,38 & 18,703 & .374 \\
\hline $\mathrm{SOA}^{\mathrm{a}}$ & 1.91 & 2,38 & 19,856 & .091 & $107.25^{* *}$ & 2,38 & 19,048 & .850 \\
\hline SOA $\times$ Trial Type & $<1$ & 2,38 & 5,901 & .048 & $5.86^{* *}$ & 4,76 & 8,145 & .236 \\
\hline \multicolumn{9}{|l|}{ Stop-R2 Condition } \\
\hline Trial Type ${ }^{c}$ & $45.27^{* *}$ & 2,38 & 8,779 & .704 & $60.53^{* *}$ & 1,19 & 9,107 & .761 \\
\hline $\mathrm{SOA}^{\mathrm{a}}$ & 1.35 & 2,38 & 9,478 & .066 & $103.23^{* *}$ & 2,38 & 6,417 & .845 \\
\hline SOA $\times$ Trial Type & $7.12^{* * *}$ & 4,76 & 3,938 & .273 & $<1$ & 2,38 & 3,526 & .048 \\
\hline
\end{tabular}

${ }^{a}$ SOA (100, 300, $\left.900 \mathrm{~ms}\right) .{ }^{\mathrm{b}}$ Trial Type (go, signal-respond) for RT1; Trial Type (go, signal-response, signal-inhibit) for RT2. ${ }^{\mathrm{c}}$ Trial Type (go, signal-response, signal-inhibit) for RT1; Trial Type (go, signalrespond) for RT2.

*** $p<.01$. 

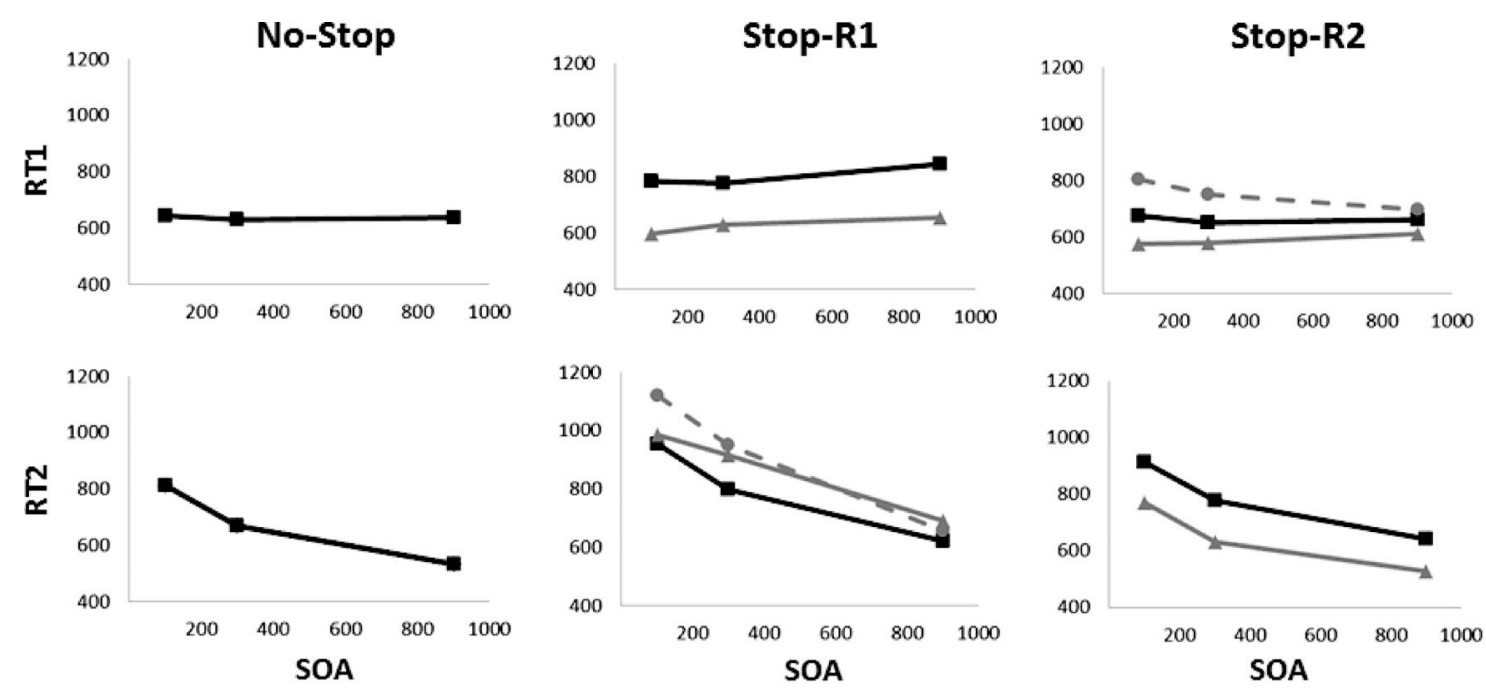

$\rightarrow$ Go $\rightarrow$ Signal-Respond $\leadsto$ Signal-Inhibit

Figure 3. RT for Task1 (RT1; top row) and Task2 (RT2; bottom row) as a function of Trial Types and SOA for the No-Stop (left column), Stop-R1 (center column), and Stop-R2 (right column) conditions of Experiment 1.

100-, 300-, and 900-ms SOAs for the Stop-R2 group, respectively. For the single-task conditions, SSD was initially set at $250 \mathrm{~ms}$ for both groups. The tracking procedure was used to adjust SSD in the same manner as in Experiment 1.

\section{Results}

Overt RT. Trials were filtered and discarded in the same manner as in Experiment 1 (1.39\%). Percentages of errors are summarized in Table 5. RT1 and RT2 were first analyzed for the dual-task trials in the same manner as in Experiment 1 (see Table 6), which is summarized in Figure 5.

In general, the dual-task conditions of Experiment 2 replicated the major results of Experiment 1. RT2 showed the PRP effect in both the Stop-R1 and Stop-R2 conditions, but RT1 was not affected much by SOA. In the Stop-R1 group, RT1 was shorter for signal-respond trials $(M=544 \mathrm{~ms})$ than for go trials $(M=671$ $\mathrm{ms})$. RT2 was longer for signal-inhibit trials $(M=772 \mathrm{~ms})$ than for go and signal-respond trials ( $M=638$ and $628 \mathrm{~ms}$, respectively). In the Stop-R2 group, RT2 was shorter for signal-respond trials $(M=548 \mathrm{~ms})$ than for go trials $(M=622 \mathrm{~ms})$. RT1 was shortest for signal-respond trials $(M=505 \mathrm{~ms})$, intermediate for go trials

Table 3

SSD and the Probability of Responding Given Signal, or $p($ Respond|Signal), for the Stop-R1 and Stop-R2 Conditions of Experiment 1 as a Function of SOA

\begin{tabular}{cccccc}
\hline & \multicolumn{2}{c}{ SSD } & & \multicolumn{2}{c}{ p(Respond|Signal) } \\
\cline { 2 - 3 } \cline { 6 - 6 } SOA & Stop-R1 & Stop-R2 & & Stop-R1 & Stop-R2 \\
\hline 100 & 371 & 486 & & .41 & .34 \\
300 & 403 & 389 & & .40 & .41 \\
900 & 435 & 327 & & .38 & .45 \\
\hline
\end{tabular}

( $M=546 \mathrm{~ms}$ ), and longest for signal-inhibit trials $(M=617 \mathrm{~ms})$. The RT1 differences in the Stop-R2 group were most pronounced at the 100-ms SOA and decreased across SOAs.

To compare the dual- and single-task conditions, RT1 in the Stop-R1 group and RT2 in the Stop-R2 group were averaged across SOAs and subjected to ANOVA as a function of Trial Type (go, signal-respond) and Condition (dual-task, single-task). The results are summarized in Table 7. Most important, RT1 was longer for the dual-task trials $(M=608 \mathrm{~ms})$ than for the singletask trials $(M=464 \mathrm{~ms})$. RT2 was also longer for the dual-task trials $(M=618 \mathrm{~ms})$ than for the single-task trials $(M=485 \mathrm{~ms})$. The outcomes indicate dual-task interference.

Stop-Signal RT. The probability of responding in stop-signal trials and mean SSD are summarized in Table 8. As intended, the probability was close to .5 in all conditions. SSRT was computed in the same manner as in Experiment 1 (see Figure 6) and submitted to ANOVA as a function of Condition (Stop-R1, Stop-R2) and SOA (100, 300, 900, single-task). The results are shown in Table 9. As in Experiment 1, neither the main effects nor the interaction approached significance. SSRT was very short for both

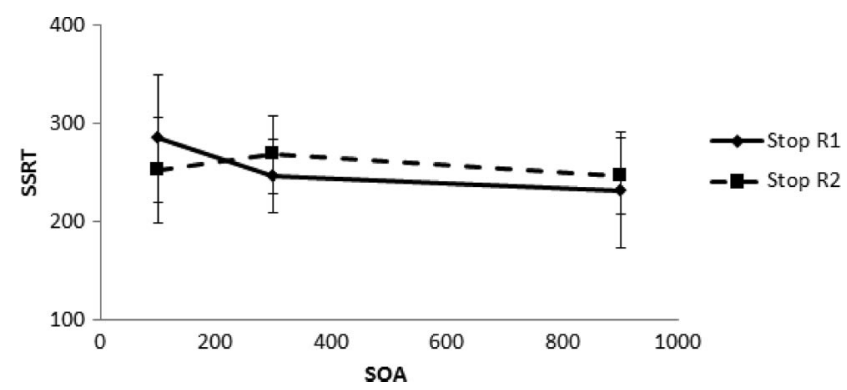

Figure 4. SSRT for the Stop-R1 and Stop-R2 conditions of Experiment 1 (the error bars represent $95 \%$ confidence intervals). 
Table 4

ANOVA for SSRT as a Function of SOA and Condition in Experiment 1

\begin{tabular}{lccrr}
\hline \multicolumn{1}{c}{ Factor } & $F$ & $d f$ & \multicolumn{1}{c}{$M S E$} & $\eta_{p}^{2}$ \\
\hline SOA $^{\mathrm{a}}$ & 1.55 & 2,38 & 5,728 & .075 \\
Condition $^{\mathrm{b}}$ & 0.75 & 2,38 & 11,757 & $<.001$ \\
SOA $\times$ condition $^{2.12}$ & 2,38 & 4,166 & .100 \\
\hline
\end{tabular}

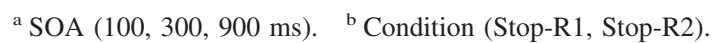

the dual-task condition $(M=235 \mathrm{~ms})$ and the single-task condition ( $M=231 \mathrm{~ms}$ ), implying no dual-task interference in the STOP process.

\section{Discussion}

The dual-task conditions of Experiment 2 replicated the main results of Experiment 1. RT2 showed the PRP effect, ranging between 220 and $480 \mathrm{~ms}$, and responses were generally faster for signal-respond trials and slower for signal-inhibit trials than for go trials. In addition, the present experiment showed that RT1 and RT2 were 130-140 ms longer in the dual-task conditions than in the single-task conditions, indicating dual-task interference. However, SSRT did not differ across SOA or between the dual- and single-task conditions. Therefore, the present experiment provides further evidence that response inhibition is not subject to dual-task interference.

\section{General Discussion}

The present study examined the influence of dual-tasking on stop-signal performance. Although previous studies have suggested that response inhibition is not affected by dual-task interference, no study directly examined stop-signal performance in a dual-task setting. Thus, the present study used a PRP task in which subjects had to inhibit one response and execute the other when a stop signal was presented.

In Experiment 1, overt responses produced large PRP effects in $\mathrm{RT} 2$, regardless of whether R1 or R2 was to be inhibited and whether inhibition was successful. In contrast, there was no refractory effect in SSRT. Previous studies showed that SSRT was unaffected by the interval between the go-task stimulus and stop signal (e.g., Logan \& Burkell, 1986), but the results of Experiment 1 provided stronger evidence that response inhibition is free from dual-task interference because response inhibition had to be performed concurrently with response execution. Furthermore, Experiment 2 compared stop-signal performance in single- and dualtask settings and showed that SSRT did not differ between these conditions. In addition, Experiment 2 replicated the null effect of SOA on SSRT in the Stop-R1 and Stop-R2 conditions, further providing support that response inhibition is independent of gotask demands.

\section{Independence of STOP and GO Processes}

The horse-race model has been a main theoretical vehicle in interpreting the results of stop-signal experiments (Logan \& Cowan, 1984). Because of its importance, studies have been con- ducted to examine the assumptions in the model (Band et al., 2003; Colonius, 1990; De Jong et al., 1990). For the most part, investigations have focused on the assumption of independence between GO and STOP processes, a violation of which might have significant influence on estimates of SSRT.

Independence of STOP and GO processes can take three forms; stochastic, contextual, and functional independence (Colonius, 1990; Logan, 1994; Verbruggen \& Logan, 2009a). Stochastic independence is a condition in which the latency of GO process (i.e., RT) is uncorrelated with the latency of STOP process (i.e., SSRT). If this assumption is false, estimates of SSRT can be biased. However, Band et al. (2003) showed that estimates of SSRT were largely unaffected by correlation between GO and STOP processes, especially when SSRTs are derived from SSDs that produce $p$ (RespondlSignal) values close to .5 (also see De Jong et al., 1990).

Contextual independence holds when the processes are invariant across different trial contexts. In particular, in a stop-signal task, GO and STOP processes are contextually independent if they are not altered by SSD and if the GO process is not altered by the presence of a stop signal (Colonius, 1990). Researchers have examined contextual independence of GO and STOP processes by determining whether mean RT for signal-respond trials exceeds RT for go trials and whether the RT distribution for signal-respond trials approaches that for go trials as SSD increases (e.g., Hanes \& Schall, 1995; Jennings et al., 1992). The outcomes of stop-signal tasks typically conform to these predictions (Logan \& Cowan, 1984), indicating behavioral validity of the contextual independence assumption.

Of most interest in the present study is functional independence between GO and STOP processes. This assumption refers to the relation between GO and STOP processes with respect to the experimental manipulations or factors that influence these processes. That is, two cognitive processes are functionally independent if factors that affect one process do not affect the other. As mentioned earlier, previous studies suggest that SSRT is virtually unaffected by the go-task demands (e.g., Logan, 1981; Logan et al., 1984), implying that STOP and GO processes depend on different experimental factors. The results of the present experiments further extend these results, demonstrating that the STOP process is not subject to the same dual-task interference as GO processes. Therefore, the horserace model assumption of functional independence between STOP and GO processes is supported.

In addition, neurophysiological and electrophysiological studies suggest that STOP and GO processes rely on different brain

Table 5

Percentages of Errors for Task1 (PE1) and Task2 (PE2) for Go Trials of the Stop-R1 and Stop-R2 Groups of Experiment 2 as a Function of SOA

\begin{tabular}{lccccc}
\hline & \multicolumn{3}{c}{ PE1 } & & \multicolumn{2}{c}{ PE2 } \\
\cline { 2 - 3 } \cline { 6 - 6 } SOA & Stop-R1 & Stop-R2 & & Stop-R1 & Stop-R2 \\
\hline Single & 2.77 & - & & - & 2.77 \\
100 & 3.31 & 2.69 & & 7.54 & 6.49 \\
300 & 3.39 & 3.77 & & 7.46 & 5.95 \\
900 & 3.39 & 2.84 & & 4.53 & 5.36 \\
\hline
\end{tabular}


Table 6

ANOVA for Overt Reaction Times for Task1 (RT1) and Task2 (RT2) for the Dual-Task

Conditions of Experiment 2

\begin{tabular}{|c|c|c|c|c|c|c|c|c|}
\hline \multirow[b]{2}{*}{ Factor } & \multicolumn{4}{|c|}{ RT1 } & \multicolumn{4}{|c|}{ RT2 } \\
\hline & $F$ & $d f$ & $M S E$ & $c_{p}^{2}$ & $F$ & $d f$ & $M S E$ & $c_{p}^{2}$ \\
\hline \multicolumn{9}{|l|}{ Stop-R1 group } \\
\hline Trial Type ${ }^{\mathrm{a}}$ & $78.54^{* *}$ & 1,19 & 6,159 & .805 & $44.99^{* *}$ & 2,38 & 8,668 & .703 \\
\hline $\mathrm{SOA}^{\mathrm{b}}$ & $<1$ & 2,38 & 6,652 & .026 & $212.94^{* *}$ & 2,38 & 8,013 & .918 \\
\hline SOA $\times$ Trial Type & $<1$ & 2,38 & 1,249 & .049 & $44.87^{* *}$ & 4,76 & 2,013 & .703 \\
\hline \multicolumn{9}{|l|}{ Stop-R2 group } \\
\hline Trial Type ${ }^{\mathrm{c}}$ & $83.98^{* * *}$ & 2,38 & 2,311 & .816 & $52.25^{* *}$ & 1,19 & 4,212 & .733 \\
\hline $\mathrm{OA}^{\mathrm{b}}$ & $20.76^{* *}$ & 2,38 & 2,600 & .522 & $135.14^{* *}$ & 2,38 & 4,191 & .877 \\
\hline SOA $\times$ Trial Type & $10.59^{* *}$ & 4,76 & 1,288 & .358 & 2.77 & 2,38 & 1,059 & .127 \\
\hline
\end{tabular}

a Trial Type (go, signal-respond) for RT1; Trial Type (go, signal-respond, signal-inhibit) for RT2. ${ }^{\text {b }}$ SOA (100, $300,900 \mathrm{~ms}$ ). $\quad{ }^{\mathrm{c}}$ Trial Type (go, signal-respond, signal-inhibit) for RT1; Trial Type (go, signal-respond) for RT2.$$
{ }^{* * *} p<.01 \text {. }
$$

structures. The STOP process appears to depend on right inferior frontal cortex (Aron \& Poldrack, 2005), basal ganglia (Band \& Boxtel, 1999; Boxtel, van der Molen, Jennings, \& Brunia, 2001), and $\mathrm{GABA}_{\mathrm{B}}$ ergic intracortical inhibitory circuit in M1 (van den Wildenberg et al., 2009), whereas the bottleneck in the GO process appears to depend on left posterior lateral prefrontal cortex (Dux, Ivanoff, Asplund, \& Marois, 2006) and left inferior frontal sulcus (Schubert \& Szameitat, 2003). Moreover, single cell recording in the frontal eye field and superior colliculi suggests that there are functionally distinct cells that initiate or inhibit saccades during saccadic countermanding tasks (e.g., Hanes, Patterson, \& Schall, 1998; Paré \& Hanes, 2003). Although neural mechanisms of stop-signal and PRP performance are not fully understood, these suggestions conform to the horse-race model.

\section{No Bottleneck in Response Inhibition?}

The majority of researchers attribute the PRP effect to temporal overlap between cognitive bottleneck processes, which might be structurally imposed (Byrne \& Anderson, 2001; Pashler, 1994; Welford, 1952) or strategically created to optimize performance (Logan \& Gordon, 2001; Meyer \& Kieras, 1997). A typical PRP model assumes that the go-task process consists of three sequential stages - perceptual, decisional, and motoric stages - in which the decisional stage constitutes the bottleneck and the remaining stages operate in parallel (e.g., Pashler, 1994; Sigman \& Dehaene, 2005). Such a model might suggest that a possible reason for the lack of dual-task interference in stop-signal performance may be because of the fact that the go-task process is interrupted before the bottleneck process starts or after the bottleneck process has completed. This would not explain the lack of dual-task interference between these points, when the bottleneck process is underway.

If response inhibition is achieved by terminating the go-task process before the bottleneck process starts (i.e., at the perceptual stage), STOP process could occupy the bottleneck without being queued and thus, produce no PRP effect. Consistent with this explanation, Logan and Burkell (1986) found that subjects who
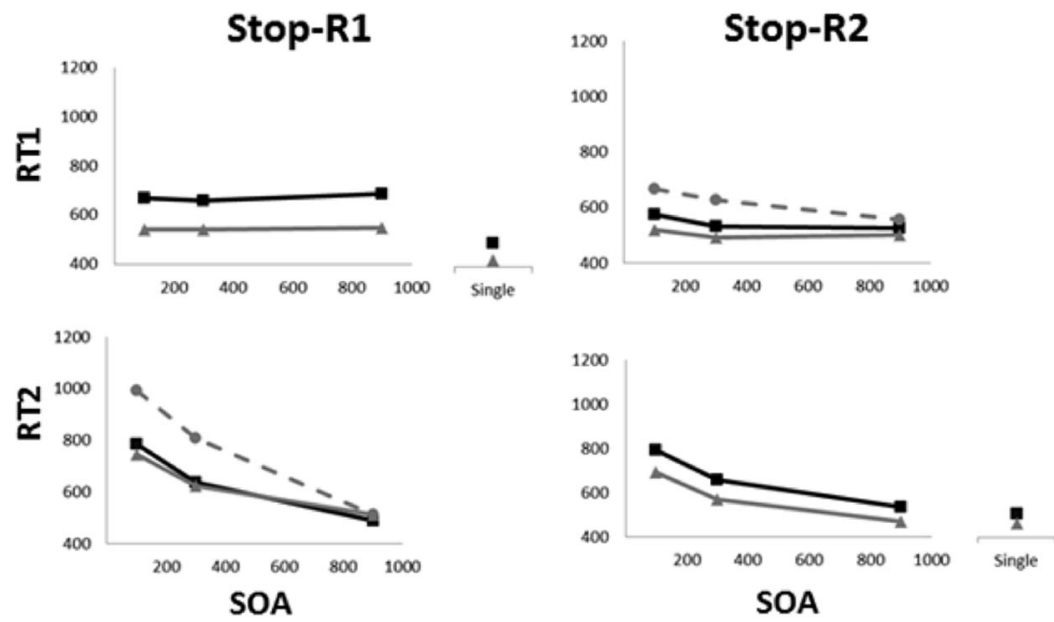

$\rightarrow$ Go $\rightarrow$ Signal-Respond $\rightarrow$ Signal-Inhibit

Figure 5. Reaction time for Task1 (RT1; top row) and Task2 (RT2; bottom row) as a function of Trial Types and SOA for the Stop-R1 (left column) and Stop-R2 (right column) groups of Experiment 2. 
Table 7

ANOVA for Overt Reaction Times for the Stop-R1 and Stop-R2 Groups of Experiment 2, Comparing the Dual-and Single-Task Conditions

\begin{tabular}{|c|c|c|c|c|}
\hline Factor & $F$ & $d f$ & $M S E$ & $\eta_{p}^{2}$ \\
\hline \multicolumn{5}{|l|}{ Stop-R1 group } \\
\hline Trial Type ${ }^{\mathrm{a}}$ & $97.20^{* * *}$ & 1,19 & 1,991 & .836 \\
\hline Condition $^{\mathrm{b}}$ & $36.13^{* *}$ & 1,19 & 11,456 & .655 \\
\hline Trial Type $\times$ Condition & $15.79^{* * *}$ & 1,19 & 1,037 & .454 \\
\hline \multicolumn{5}{|l|}{ Stop-R2 group } \\
\hline Trial Type ${ }^{\mathrm{a}}$ & $38.03^{* * *}$ & 1,19 & 2,173 & .667 \\
\hline Condition $^{\mathrm{b}}$ & $94.00^{* * *}$ & 1,19 & 3,818 & .832 \\
\hline Trial Type $\times$ Condition & $9.01^{* *}$ & 1,19 & 1,013 & .322 \\
\hline
\end{tabular}

${ }^{\mathrm{a}}$ Trial Type (go, signal-respond). $\quad{ }^{\mathrm{b}}$ Condition (dual-task, single-task). **** $p<.01$.

responded to tones by withholding the go-task response and executing another keypress (stop-change task) showed a PRP effect in responses to the tones when they failed to inhibit the go-task response (i.e., signal-respond trials) but not when they successfully inhibited the go-task response (signal-inhibit trials). However, this explanation does not apply to the present results. In Stop-R1 conditions of the two experiments, RT2 still produced the PRP effect when R1 was successfully inhibited. Thus, inhibition of R1 did not occur before the bottleneck process.

In addition, the lack of dual-task interference in SSRT in the Stop-R2 condition contradicts this prebottleneck termination of the GO process: At short SOAs, the bottleneck is occupied by GO1 when GO2 requires it, yielding the PRP effect. Consequently, if the STOP process involved the bottleneck processing, it could avoid being queued only if the STOP process had started before the bottleneck in GO1 started. SSDs obtained in the present experiments ranged from 50 to $60 \%$ of the corresponding RT2s, which are too long for this condition to be satisfied.

Response inhibition could also be achieved by terminating the GO process only after its bottleneck process has completed (at the motoric stage). Nothing would have prevented this from happening in the Stop-R2 condition. However, this possibility is unlikely in the Stop-R1 condition because the bottleneck should be occupied by GO2 after the bottleneck in GO1 completes, so STOP can start only after $\mathrm{GO} 2$ has released the bottleneck, which would be too late to inhibit R1. It is possible that STOP arrives at the bottleneck before GO2 does, so STOP starts the bottleneck processing soon after GO1 releases it and before GO2 starts it, which would allow

Table 8

SSD and the Probability of Responding Given Signal, or p(Respond|Signal), for the Stop-R1 and Stop-R2 Groups of Experiment 2 as a Function of SOA

\begin{tabular}{lcccccc}
\hline & \multicolumn{2}{c}{ SSD } & & \multicolumn{2}{c}{ p(Respond|Signal) } \\
\cline { 2 - 3 } SOA & Stop-R1 & Stop-R2 & & Stop-R1 & Stop-R2 \\
\hline Single & 240 & 241 & & .49 & .50 \\
100 & 380 & 500 & & .48 & .52 \\
300 & 391 & 399 & & .49 & .51 \\
900 & 390 & 272 & & .49 & .52 \\
\hline
\end{tabular}

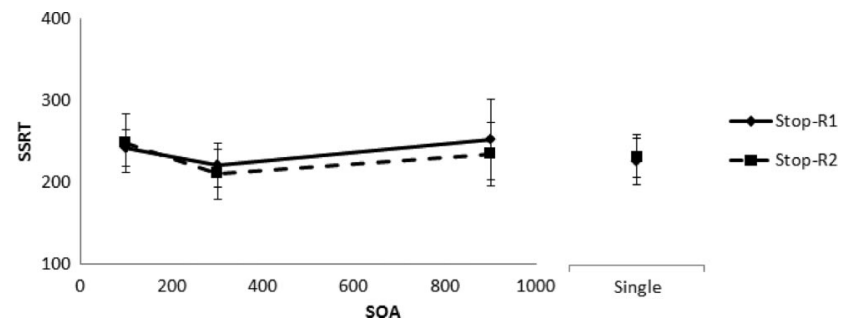

Figure 6. SSRT for the Stop-R1 and Stop-R2 groups of Experiment 2 (the error bars represent $95 \%$ confidence intervals).

postbottleneck termination of GO1. This scenario seems viable at long SOAs when GO2 starts late. However, at short SOAs, this might not happen, as the present data suggest. SSD was about 400 $\mathrm{ms}$ in the Stop-R1 condition, which was $300 \mathrm{~ms}$ after S2 onset at the 100-ms SOA. Given that simple RT typically ranges between 150 and $250 \mathrm{~ms}$ (e.g., Luce, 1986), the prebottleneck process should have completed before stop-signal onset, which allows GO2 to occupy the bottleneck before STOP arrives. Therefore, it is difficult to attribute the lack of dual-task interference in SSRT to pre- or postbottleneck termination of the GO processes.

Another possibility is that the bottleneck process in response inhibition is "latent" (Ruthruff et al., 2003). Previous studies demonstrated that the PRP effect can be eliminated in very specific conditions; subjects are extensively trained with tasks and stimuli are mapped to highly compatible responses (Hazeltine et al., 2002; Schumacher et al., 2001). These two factors are assumed to produce a situation in which the bottleneck process (i.e., response selection) completes very rapidly so that there is a minimum amount of temporal overlap between bottleneck processes (Anderson, Taatgen, \& Byrne, 2005; Ruthruff et al., 2003). Thus, it is possible that response inhibition involves a bottleneck process but the process is very brief (latent) so that the PRP effect did not emerge at the surface. In accord with this explanation, Boucher, Palmeri, Logan, and Schall (2007) developed an interactive race model for a saccade countermanding task in which the GO and STOP processes interact only for a brief period near the end of SSRT. A limitation is that Boucher et al.'s model has only been fitted to the data of a saccade task. Thus, it remains to be seen whether the modeling results can be generalized to other task conditions with manual responses.

Finally, it should be noted that some previous PRP studies (e.g., De Jong, 1993; Smith, 1967) used go/no-go task as Task1 and found the PRP effect in RT2 even when R1 was not executed (i.e., on no-go trials). Such outcomes might be interpreted as indicating that Task 2 process was queued until inhibition of R1 completed.

Table 9

ANOVA for SSRT as a Function of SOA and Condition in Experiment 2

\begin{tabular}{lllrl}
\hline \multicolumn{1}{c}{ Factor } & \multicolumn{1}{c}{$F$} & \multicolumn{1}{c}{$d f$} & MSE & $\eta_{p}^{2}$ \\
\hline SOA $^{\mathrm{a}}$ & 2.32 & 3,114 & 3,236 & .057 \\
Condition $^{\mathrm{b}}$ & $<1$ & 1,38 & 10,580 & .002 \\
SOA $\times$ Condition & $<1$ & 3,114 & 3,236 & .011 \\
\hline
\end{tabular}

${ }^{\mathrm{a}} \mathrm{SOA}$ (100, 300, $900 \mathrm{~ms}$, single-task). $\quad{ }^{\mathrm{b}}$ Condition (Stop-R1, Stop-R2). 
This would imply functional dependence of GO and STOP processes if no-go trials are assumed to involve the same kind of inhibitory process as in the stop-signal procedure (e.g., Bissett, Nee, \& Jonides, 2009; Friedman \& Miyake, 2004). However, that assumption may not be valid. Gomez, Ratcliff, and Perea (2007) developed diffusion models for go/no-go tasks and found that a model that had response boundaries for go and no-go responses fit data better than a model that had a boundary only for go response, suggesting that decision process in go/no-go tasks is better characterized as choice between alternative responses, as in typical choice-reaction tasks.

In addition, there is evidence that presentation of a stimulus associated with a no-go response prolongs go RT but has no effect on SSRT (Bissett et al., 2009), implying that the stimulus automatically activated an implicit no-go response without activating inhibitory process. Thus, even if the go/no-go and stop-signal paradigms do involve the same type of inhibitory processes, the PRP effect in overt response after no-go trials may be because of the response-selection process involved in go/no-go tasks, not the inhibitory process per se. This suggests that SSRT might be subject to the PRP effect when a selective component is introduced in the STOP process (i.e., selective stopping). This possibility needs to be pursued in future studies.

\section{Influence of Task Prioritization in Stop-Signal Tasks}

A major difference between the PRP and stop-signal paradigms may be how the two responses are prioritized. ${ }^{2}$ It is known that performance of two concurrent tasks trades off according to which task is prioritized (see Navon \& Gopher, 1979). In some PRP studies, experimenters explicitly instruct subjects to prioritize Task1 and then shift to Task2 at completion of the former. Many of recent PRP studies exclude this explicit prioritization of Task1 (Levy \& Pashler, 2001), but the emphasis on response speed in task instructions may implicitly require subjects to prioritize Task1 over Task2 when S1 is always (or almost always) presented first in an experiment (but see, Sigman \& Dehaene, 2006, for the PRP effect with a random task order). In such conditions, there is little or no effect of SOA on RT1, implying the importance of task prioritization. In fact, when two tasks are equally emphasized in instructions, dual-task interference can be eliminated (e.g., Schumacher et al., 2001; but see Levy \& Pashler, 2001). This might explain the lack of dual-task interference in STOP process in the present study; that is, subjects might have prioritized stopping over going and protected the former process from interference (Logan, 1985).

In stop-signal tasks, instructions usually do not prioritize stopping over going. Instructions emphasize the importance of maintaining fast and accurate responding in the go task rather than stopping. This emphasis is placed to ensure that subjects do not delay go responses to wait for a stop signal. In addition, when a tracking procedure is used, subjects are often told that SSD will be adjusted so they should expect successful stopping only on half the trials. Moreover, stop signals typically occur on a minority of the trials (20-30\%; Logan, 1994; Logan \& Cowan, 1984; Verbruggen \& Logan, 2008), whereas the go task occurs on every trial. When the proportion of stop-signal trials increases, go RT increases, suggesting a shift in priority toward stopping, yet SSRT is unaffected by this manipulation (Bissett \& Logan, 2011; Logan, 1981;
Logan \& Burkell, 1986; Verbruggen \& Logan, 2009b), suggesting that STOP process is insensitive to prioritization. Furthermore, Experiment 2 of the present study showed significant dual-task interference in RT1 as compared to the single-task condition, which indicates that prioritizing Task 1 in the dual-task condition did not protect it from interference. Thus, prioritizing the STOP process may not protect it from interference either. Collectively, these findings suggest that it is not the prioritization of response inhibition that is responsible for the lack of dual-task interference in SSRT.

\section{Dual-Task Interference in Stopping Continuous Actions?}

The present study focused on dual-task interference in discrete actions because they are most often used in psychological studies. Some studies suggest that response inhibition might operate differently in continuous actions. For instance, Williams (1971) had subjects swing their arm and reverse it in response to a signal. They found that the time to decelerate the arm swing increased with decreasing SOA. Vince (1950; cited in Welford, 1952) also obtained a PRP-like effect in the time required to stop manual tracking, and Vince and Welford (1967) found delays in slowing down line drawing for short SOAs (but no effect on speeding up the drawing). These observations suggest that there is a refractory effect in stopping time when the task requires continuous movement.

However, other studies reported contrary results. Gottsdanker (1966) used a task similar to Vince's and found little effect of SOA on corrective manual movements. Logan (1982) studied stopsignal performance in typewriting and found that the number of letters typed after a stop signal decreased slightly when SOA decreased, yielding a trend in the opposite direction of the PRP effect. More recently, Horstmann (2003) obtained a PRP-like effect in stopping continuous finger tapping, but only when tapping was initiated by a go signal immediately preceding a stop signal, not when it was a continuation of tapping that had been initiated earlier. Horstmann argued that there may be differences between inhibition of an ongoing action and inhibition of the initiation of an action (but others found little evidence for such distinction; e.g., Logan, 1982; Morein-Zamir et al., 2004).

Hence, studies of stopping continuous actions provide mixed results. It is not clear whether stopping of continuous actions suffers from dual-task interference or involves different mechanisms than those involved in stopping discrete actions. More important, no study has examined stopping of continuous actions in dual-task settings. Therefore, future investigations are needed to examine the extent to which the present results are generalized to stopping of continuous actions.

\section{Conclusion}

The present study examined stop-signal performance in dualtask contexts. Two main observations were that (a) SSRT did not show a PRP effect and that (b) SSRT did not differ between dualand single-task conditions. The first observation reinforces a sim-

\footnotetext{
${ }^{2}$ We thank an anonymous reviewer for raising this issue
} 
ilar conclusion by Logan and Burkell (1986) study and provides stronger evidence because response inhibition had to be performed without inhibiting another response in the present study. The second observation extends several previous findings (e.g., Logan, 1981; Logan et al., 1984) that response inhibition is independent of the go-task demands, implying that additional task demands do not influence the STOP process. These results indicate that response inhibition in the stop-signal paradigm does not suffer dual-task interference, at least when responses are discrete actions. Therefore, the present study supports the horse-race assumption of functional independence between the STOP and GO processes.

\section{References}

Anderson, J. R., Taatgen, N. A., \& Byrne, M. D. (2005). Learning to achieve perfect timesharing: Architectural implications of Hazeltine, Teague, and Ivry (2002). Journal of Experimental Psychology: Human Perception and Performance, 31, 749-761.

Aron, A. R., \& Poldrack, R. A. (2005). The cognitive neuroscience of response inhibition: Relevance for genetic research in attention-deficity/ hyperactivity disorder. Biological Psychiatry, 57, 1285-1292.

Band, G. P. H., \& Boxtel, G. J. M. (1999). Inhibitory motor control in stop paradigms: Review and reinterpretation of neural mechanisms. Acta Psychologica, 101, 179-211.

Band, G. P. H., van der Molen, M. W., \& Logan, G. D. (2003). Horse-race model simulations studies of the stop signal procedure. Acta Psychologica, 112, 105-142.

Bissett, P. G., \& Logan, G. D. (2011). Balancing cognitive demands: Control adjustments in the stop-signal paradigm. Journal of Experimental Psychology: Learning, Memory, and Cognition, 37, 392-404.

Bissett, P. G., Nee, D. E., \& Jonides, J. (2009). Dissociating interferencecontrol processes between memory and response. Journal of Experimental Psychology: Learning, Memory, and Cognition, 35, 1306-1316.

Boucher, L., Palmeri, T. J., Logan, G. D., \& Schall, J. D. (2007). Inhibitory control in mind and brain: An interactive model of countermanding saccades. Psychological Review, 114, 376-397.

Boxtel, G. J. M., van der Molen, M. W., Jennings, J. R., \& Brunia, C. H. M. (2001). A psychophysiological analysis of inhibitory motor control in the stop-signal paradigm. Biological Psychology, 58, 229-262.

Byrne, M. D., \& Anderson, J. R. (2001). Serial modules in parallel: The psychoogical refractory period and perfect time-sharing. Psychological Review, 108, 847-869.

Chamberlain, S. R., Fineberg, N. A., Blackwell, An, D., Robbins, T. W., \& Sahakian, B. J. (2006). Motor inhibition and cognitive flexibility in obsessive-compulsive disorder and trichotillomania. American Journal of Psychiatry, 163, 1282-1284.

Colonius, H. (1990). A note on the stop-signal paradigm, or how to observe the unobservable. Psychological Review, 97, 309-312.

De Jong, R. (1993). Multiple bottlenecks in overlapping task performance. Journal of Experimental Psychology: Human Perception and Performance, 19, 965-980.

De Jong, R., Coles, M. G. H., Logan, G. D., \& Gratton, G. (1990). Searching for the point of no return: The control of response processes in speeded choice reaction performance. Journal of Experimental Psychology: Human Perception and Performance, 16, 164-182.

Dux, P. E., Ivanoff, J., Asplund, C. L., \& Marois, R. (2006). Isolation of a central bottleneck of information processing with time-resolving fMRI. Neuron, 52, 1109-1120.

Enticott, P. G., Ogloff, J. R. P., \& Bradshaw, J. L. (2008). Response inhibition and impulsivity in schizophrenia, Psychiatry Research, 157, 251-254.

Friedman, N. P., \& Miyake, A. (2004). The relations among inhibition and interference control functions: A latent-variable analysis. Journal of Experimental Psychology: General, 133, 101-135.
Gomez, P., Ratcliff, R., \& Perea, M. (2007). A model of the go/no-go task Journal of Experimental Psychology: General, 136, 389-413.

Gottsdanker, R. (1966). The effect of superseding signals. Quarterly Journal of Experimental Psychology, 18, 236-249.

Gottsdanker, R., \& Stelmach, G. E. (1971). The persistence of psychological refractoriness. Journal of Motor Behavior, 3, 301-312.

Gray, R. (2009). A model of motor inhibition for a complex skill: Baseball batting. Journal of Experimental Psychology: Applied, 15, 91-105.

Greenwald, A. G., \& Shulman, H. (1973). On doing two things at once: II. Elimination of the psychological refractory period. Journal of Experimental Psychology, 101, 70-76.

Hanes, D. P., Patterson, W. F. II, \& Schall, J. D. (1996). Role of frontal eye fields in countermanding saccades: Visual, movement, and fixation activity. Neurophysiology, 79, 817-834.

Hanes, D. P., \& Schall, J. D. (1995). Countermanding saccades in macaque. Visual Neuroscience, 12, 929-937.

Hasher, L., \& Zacks, R. T. (1988). Working memory, comprehension, and aging: A review and a new view. In G. H. Bower (Ed.), The Psychology of Learning and Motivation (Vol. 22, pp. 193-225). New York: Academic Press.

Hazeltine, E., Teague, D., \& Ivry, R. B. (2002). Simultaneous dual-task performance reveals parallel response selection after practice. Journal of Experimental Psychology: Human Perception and Performance, 28, $527-545$.

Horstmann, G. (2003). The psychological refractory period of stopping Journal of Experimental Psychology: Human Perception and Performance, 29, 956-981.

Jennings, J. R., van der Molen, M. W., Brock, K., \& Somsen, R. J. M. (1992). On the synchrony of stopping motor responses and delaying heartbeats. Journal of Experimental Psychology: Human Perception and Performance, 18, 422-436.

Lappin, J. S., \& Eriksen, C. W. (1966). Use of a delayed signal to stop a visual reaction-time response. Journal of Experimental Psychology, 72, 805-811.

Levy, J., \& Pashler, H. (2001). Is dual-task slowing instruction dependent? Journal of Experimental Psychology: Human Perception and Performance, 27, 862-869.

Lien, M.-C., \& Proctor, R. W. (2002). Stimulus-response compatibility and psychological refractory period effects: Implications for response selection. Psychonomic Bulletin \& Review, 9, 212-238.

Logan, G. D. (1981). Attention, automaticity, and the ability to stop a speeded choice response. In J. Long \& A. D. Baddeley (Eds.), Attention and performance IX (pp. 205-222). Hillsdale, NJ: Erlbaum.

Logan, G. D. (1982). On the ability to inhibit complex actions: A stopsignal study of typewriting. Journal of Experimental Psychology: Human Perception and Performance, 8, 778-792.

Logan, G. D. (1985). Executive control of thought and action. Acta Psychologica, 60, 193-210.

Logan, G. D. (1994). On the ability to inhibit thought and action: A users' guide to the stop signal paradigm. In D. Dagenbach \& T. H. Carr (Eds.), Inhibitory processes in attention, memory, and language. (pp. 189-239). San Diego: Academic Press.

Logan, G. D., \& Burkell, J. (1986). Dependence and independence in responding to double stimulation: A comparison of stop, change, and dual-task paradigms. Journal of Experimental Psychology: Human Perception and Performance, 12, 549-563.

Logan, G. D., \& Cowan, W. B. (1984). On the ability to inhibit thought and action: A theory of an act of control. Psychological Review, 91, 295327.

Logan, G. D., Cowan, W. B., \& Davis, K. A. (1984). On the ability to inhibit responses in simple and choice reaction time tasks: A model and a method. Journal of Experimental Psychology: Human Perception and Performance, 10, 276-291. 
Logan, G. D., \& Gordon, R. D. (2001). Executive control of visual attention in dual-task situations. Psychological Review, 108, 393-434.

Logan, G. D., Schachar, R. J., \& Tannock, R. (2000). Executive control problems in childhood psychopathology: Stop-signal studies of attention deficit disorder. In S. Monsell \& J. Driver (Eds.), Attention and performance XVIII. (pp. 653-677). Cambridge MA: MIT Press.

Luce, R. D. (1986). Response times. New York: Oxford University Press. Meyer, D. E., \& Kieras, D. E. (1997). A computational theory of executive cognitive processes and multiple-task performance: Pt. 2. Accounts of psychological refractory-period phenomena. Psychological Review, 104, 749-791.

Moren-Zamir, S., Nagelkerke, P., Chua, R., Franks, I., \& Kingstone, A. (2004). Inhibiting prepared and ongoing responses: Is there more than one kind of stopping? Psychonomic Bulletin \& Review, 11, 1034-1040.

Navon, D., \& Gopher, D. (1979). On the economy of the human-processing system. Psychological Review, 86, 214-255.

Nelson, M. J., Boucher, L., Logan, G. D., Palmeri, T. J., \& Schall, J. D. (2010). Nonindependent and nonstationary response times in stopping and stepping saccade tasks. Attention, Perception \& Psychophysics, 72, 1913-1929.

Ollman, R. T. (1973). Simple reactions with random countermanding of the "go" signal. In S. Kornblum (Ed.), Attention and performance IV (pp. 571-581). New York: Academic Press.

Paré, M., \& Hanes, D. P. (2003). Controlled movement processing: Superior colliculus activity associated with countermanding saccades. Journal of Neuroscience, 23, 6480-6489.

Pashler, H. (1994). Dual-task interference in simple tasks: Data and theory. Psychological Bulletin, 16, 220-244.

Rieger, M., \& Gauggel, S. (1999). Inhibitory after-effects in the stop signal paradigm. British Journal of Psychology, 90, 509-518.

Ruthruff, E., Johnston, J. C., \& Van Selst, M. (2001). Why practice reduces dual-task interference? Journal of Experimental Psychology: Human Perception and Performance, 27, 3-21.

Ruthruff, E., Johnston, J. C., Van Selst, M., Whitsell, S., \& Remington, R. (2003). Vanishing dual-task interference after practice: Has the bottleneck been eliminated or is it merely latent? Journal of Experimental Psychology: Human Perception and Performance, 29, 280-289.

Schubert, T., \& Szameitat, A. J. (2003). Functional neuroanatomy of interference in overlapping dual tasks: An fMRI study. Cognitive Brain Research, 17, 733-746.

Schumacher, E. H., Seymour, T. L., Glass, J. M., Kieras, D. E., \& Meyer, D. E. (2001). Virtually perfect time sharing in dual-task performance: Uncorking the central attentional bottleneck. Psychological Science, 12, 101-108.

Shin, Y. K., Cho, Y. S., Lien, M.-C., \& Proctor, R. W. (2007). Is the psychological refractory period effect for ideomotor compatible tasks eliminated by speed-stress instructions? Psychological Research, 71, $553-567$
Sigman, M., \& Dehaene, S. (2005). Parsing a cognitive task: A characterization of the mind's bottleneck. PLoS Biology, 3, e37.

Sigman, M., \& Dehaene, S. (2006). Dynamics of the central bottleneck: Dual-task and task uncertainty. PLoS Biology, 4, e220.

Smith, M. C. (1967). The psychological refractory period as a function of performance of a first response. Quarterly Journal of Experimental Psychology, 19, 350-352.

Telford, C. W. (1931). The refractory phase of voluntary and associative responses. Journal of Experimental Psychology, 14, 1-36.

Tombu, M., \& Jolicœur, P. (2003). Virtually no evidence for virtually perfect time-sharing. Journal of Experimental Psychology: Human Perception and Performance, 30, 795-810.

van den Wildenberg, W. P. M., Burle, B., Vidal, F., van der Molen, M. W., Ridderinkhof, K. R., \& Hasbroucq, T. (2009). Mechanisms and dynamics of cortical motor inhibition in the stop-signal paradigm: A TMS study. Journal of Cognitive Neuroscience, 22, 225-239.

Van Selst, M., Ruthruff, E., \& Johnston, J. C. (1999). Can practice eliminate the psychological refractory period effect? Journal of Experimental Psychology: Human Perception \& Performance, 25, 1268 1283.

Verbruggen, F., \& Logan, G. D. (2008). Response inhibition in the stopsignal paradigm. Trends in Cognitive Sciences, 12, 418-424.

Verbruggen, F., \& Logan, G. D. (2009a). Models of response inhibition in the stop-signal and stop-change paradigms. Neuroscience and Biobehavioral reviews, 33, 647-661.

Verbruggen, F., \& Logan, G. D. (2009b). Proactive adjustments of response strategies in the stop-signal paradigm. Journal of Experimental Psychology: Human Perception and Performance, 35, 835-854.

Verbruggen, F., Logan, G. D., Liefooghe, B., \& Vandierendonck, A. (2008). Aftereffects of response inhibition: Repetition priming or between-trial control adjustments? Journal of Experimental Psychology: Human Perception and Performance, 34, 413-426.

Vince, M. A. (1950). Some exceptions to the psychological refractory period in unskilled manual responses. Medical Research Council Applied Psychology Unit Report No. 124/50.

Vince, M. A., \& Welford, A. T. (1967). Time taken to change the speed of a response. Nature, 213, 532-533.

Way, T. C., \& Gottsdanker, R. (1968). Psychological refractoriness with varying differences between tasks. Journal of Experimental Psychology, $78,38-45$.

Welford, A. T. (1952). The "psychological refractory period" and the timing of high speed performance: A review and a theory. British Journal of Psychology, 43, 2-19.

Williams, L. R. T. (1971). Refractoriness of an extended movement to directional change. Journal of Motor Behavior, 3, 289-300.

Received January 6, 2011

Revision received March 16, 2011

Accepted April 7, 2011 